\title{
Risk of agranulocytosis and aplastic anaemia in relation to use of antithyroid drugs
}

\author{
International Agranulocytosis and Aplastic Anaemia Study
}

\begin{abstract}
The relation of the use of antithyroid drugs to the risk of developing agranulocytosis and aplastic anaemia was evaluated in a population based casecontrol study with patients from Israel and seven regions in Europe. Data were obtained from cases and hospital controls by interview. Use of antithyroid drugs in the week before the onset of illness was compared in 262 patients with agranulocytosis and 1771 controls. Forty five patients $(17 \%)$ and five controls $(0 \cdot 3 \%)$ had used antithyroid drugs. The relative risk was estimated to be $102(95 \%$ confidence interval 38 to 275) taking into account confounding by other factors, including the use of other drugs. The excess risk for use of antithyroid drugs in any one week was estimated to be 6.3 cases of agranulocytosis per million users. Use of antithyroid drugs in a five month period ending one month before admission to hospital was compared in 135 patients with aplastic anaemia and 2145 controls. Four patients (3\%) and five controls $(0 \cdot 2 \%)$ had taken drugs; the estimate of relative risk was $9.2(95 \%$ confidence interval 1.8 to 47 ) after control for confounding.
\end{abstract}

The estimate of excess risk of agranulocytosis with the use of antithyroid drugs was lower than found previously. Although the excess risk for aplastic anaemia was not calculated, these data suggest that it is very low.

\section{Introduction}

Antithyroid drugs have been used for over four decades, after early reports of the antithyroid effects of to the use of antithyroid drugs is thought to be rare. Agranulocytosis, however, is a well known adverse effect of antithyroid drugs and is perhaps one of the best known side effects of drugs affecting the haemopoietic system. Despite this there are few quantitative estimates of the risk of agranulocytosis and none of the risk of aplastic anaemia. ${ }^{3.5}$ We examined the relation between antithyroid drugs and both agranulocytosis and aplastic anaemia.

The data were obtained in the international agranulocytosis and aplastic anaemia study.$^{67}$ Briefly, this case-control study was conducted in Israel, Barcelona, Ulm, West Berlin, Milan, Budapest, Sofia, and Stockholm-Uppsala and had a population base of 23 million. After a pilot phase data collection began in July 1980. This report is based on cases identified up to 30 June 1986.

\section{International \\ Agranulocytosis and Aplastic Anaemia Study \\ Participants in the study are listed at the end of this paper. Manuscript prepared by: George Retsagi, Judith P} Kelly, David W Kaufman

Correspondence to: Dr David W Kaufman, Slone Epidemiology Unit, Boston University School of Medicine, Brookline, Massachusetts 02146, USA.

Patients with either dyscrasia were identified by means of telephone networks that included all hospitals in the eight regions. The diagnosis was confirmed by a panel of haematologists, who did not know whether patients had been exposed to drugs. Excluded patients comprised all those receiving chemotherapy, radiotherapy, or immunosuppressive treatment; those with systemic diseases that could be associated with neutropenia-for example, infectious mononucleosis and systemic lupus erythematosus; those with neosulphonamides and thioureas. ${ }^{12}$ Aplastic anaemia due

\section{Methods}

plastic or granulomatous disease affecting the bone marrow; and those with other conditions masquerading as either dyscrasia.

\section{Agranulocytosis}

We report only on patients who were admitted to hospital with agranulocytosis, not those who developed it while already in hospital for other conditions. To be accepted as having agranulocytosis each patient had to be at least 2 years old and to have a granulocyte count of $\leqslant 0.5 \times 10^{9} / 1$, a haemoglobin concentration of $\geqslant 100$ $\mathrm{g} / \mathrm{l}$ or a packed cell volume of $\geqslant 0 \cdot 3$, and a platelet count of $\geqslant 100 \times 10^{9} / 1$. When a bone marrow aspirate or biopsy specimen was not available the diagnosis was accepted if the neutrophil count had returned to normal within 30 days. The day of clinical onset was deemed to be the day on which sore throat, fever, or chills first occurred, as reported by the patient.

Of the 362 patients confirmed as having agranulocytosis, 67 (19\%) were not interviewed because: they had died; more than 28 days had elapsed since admission to hospital; there were psychological or medical reasons; or they could not be traced. In addition, eight patients (or their doctors) refused to participate $(2 \%)$. Forty two of the remaining 287 cases were excluded because the day of onset of their symptoms was unknown or was more than 21 days before admission, too long for detailed information about drug use during the week before onset to be reliable. Seventeen patients identified in the pilot phase of the study were included in the analysis, giving a total of 262 cases of agranulocytosis.

\section{Aplastic anaemia}

To be accepted as having aplastic anaemia a patient had to meet at least two of the following criteria: a white cell count of $\leqslant 3 \cdot 5 \times 10^{9} / 1$, a platelet count of $\leqslant 50$ $\times 10^{9} / 1$ and a haemoglobin concentration of $\leqslant 100 \mathrm{~g} / \mathrm{l}$, or a packed cell volume of $\leqslant 0.3$ with a reticulocyte count of $\leqslant 30 \times 10^{9} / 1$. Findings on bone marrow biopsy had to be compatible with the diagnosis. Nine patients for whom a biopsy specimen was not available were accepted as having the disease because at least two bone marrow aspirates showed typical signs and the clinical presentation and progression were typical of aplastic anaemia. Because the initial symptoms are vague and progression is insidious the time of onset of the disease could not be determined.

Of the 188 patients confirmed as having the disease $57(30 \%)$ could not be interviewed for the same reasons as for the patients with agranulocytosis, and one patient refused to participate. The remaining 130 patients were combined with five from the pilot study, giving a total of 135 patients for analysis.

\section{CONTROLS}

Potential controls were identified without knowledge 
trauma it was the day of the accident; for those with acute conditions it was the day on which symptoms started; for those with longstanding conditions (such as inguinal hernia) it was the day of admission. Controls whose date of onset of symptoms was known and was within 21 days of admission were included, giving 1771 controls for comparison with the patients with agranulocytosis. As the day of onset could not be determined for aplastic anaemia all 2145 controls were included for this comparison.

\section{DATA COLLECTION}

A standard questionnaire was administered by nurses and physicians trained in its use. Detailed information was obtained about use of drugs day by day for the first seven days before admission, week by week for the three weeks before that, at any time in the five months before that, and at any time in the more distant past. Patients were questioned about use for a list of indications, and a list of generic and trade names of commonly used drugs was read to them. Patients were asked if they had taken drugs for a list of thyroid disorders, and the generic and trade names of drugs commonly used to treat the disorders was read to them. To further minimise memory loss patients were interviewed only if less than 28 days had elapsed since admission. The questionnaire also included demographic and medical information and some information on exposure to radiation, chemicals, and insecticides. Information on the clinical course of illness was recorded for all subjects. Laboratory data, blood smears, bone marrow aspirates, and biopsy sections were obtained whenever possible.

\section{DEFINITION OF EXPOSURE}

The antithyroid drugs evaluated were methimazole, carbimazole, and propylthiouracil.

Agranulocytosis-Exposure to antithyroid drugs was defined as use any time in the week before the day of onset, as agranulocytosis induced by antithyroid drugs is an immunological phenomenon of fairly rapid onset and could not have been provoked if the drugs or their metabolites were no longer present in the body. ${ }^{8.11}$ Only one case (but no control) was last exposed to antithyroid drugs within the month before the day of onset but not during the last week of that month.

Aplastic anaemia-As it was not possible to time the onset of aplastic anaemia, drug exposures were defined in relation to the day of admission. Only drug use 29-180 days before admission was considered. Use confined to the month before admission was ignored because this was probably after the onset of aplastic anaemia; use confined to the period more than six months before admission was judged not to be relevant to the cause of the disease.

\section{DATA ANALYSIS}

Relative risks for categories of exposure to drugs were estimated by multiple logistic regression ${ }^{12}$ to control simultaneously for all potential confounding factors, including exposure to other drugs.

For agranulocytosis the multiple logistic regression model included terms for the following factors in addition to use of antithyroid drugs: age; sex; geographical region; date of interview; reliability of the patient (as judged by the interviewer); person interviewed (patient, parent, or guardian); transfer from another hospital; history of allergy, blood disease, or infectious mononucleosis; exposure to insecticides; and use of sulphonamides and other anti-infective drugs, dipyrone, butazones, indomethacin, other "suspect" drugs, and other drugs in the week before the day of onset.

For aplastic anaemia the model included, in addition to use of antithyroid drugs: age; sex; geographical region; date of interview; reliability of the patient; person interviewed; transfer from another hospital; history of blood disease and tuberculosis; history of exposure to benzene, insecticides, and petrochemicals; and use of drugs (sulphonamides, other anti-infective drugs, diclofenac, butazones, indomethacin, other suspect drugs, and other drugs) during days 29-180 before admission.

Census data from the eight regions were used to estimate the average overall yearly incidence of each dyscrasia. Not all centres participated for the full period of six years, and appropriate adjustments were made in estimating annual rates. The annual incidence of agranulocytosis was 3.9 patients admitted to hospital per million and of aplastic anaemia $2 \cdot 2$ patients per million. The proportion of cases associated with the use of antithyroid drugs was calculated ${ }^{1314}$ and used together with the incidence and the estimate of relative risk to estimate the excess risk. ${ }^{15-17}$

\section{Results}

AGRANULOCYTOSIS

Forty five patients $(17 \%)$ with agranulocytosis and five controls $(0.3 \%)$ reported taking antithyroid drugs in the week before the day of onset. The multivariate estimate of relative risk comparing use of antithyroid drugs with no use during that period was $102(95 \%$ confidence interval 38 to 275). Because of the small number of controls, relative risks specific to regions were not estimated.

The proportion of cases due to antithyroid drugs was calculated to be $17 \%$. (When the relative risk is large this fraction is almost identical with the proportion of exposed cases.) This fraction, the average incidence of agranulocytosis in one week, and the relative risk for use of antithyroid drugs in the week before the day of onset were used to calculate the excess risk for the use of antithyroid drugs, which was $6 \cdot 3$ cases per million users for any exposure in a one week period.

In over $90 \%$ of cases and all controls using antithyroid drugs the drugs were taken daily and continued to be used up to the day of onset (table). Thirty seven

Use of antithyroid drugs by patients with agranulocytosis $(n=45)$ and controls $(n=5)$ and by patients with aplastic anaemia $(n=4)$ and controls $(n=5)$

\begin{tabular}{|c|c|c|}
\hline & Cases & Controls \\
\hline & No $(\%)$ & No $(\%)$ \\
\hline \multicolumn{3}{|c|}{ Agranulocytosis } \\
\hline Drug used daily & $44(98)$ & $5(100)$ \\
\hline Dr st used on day before onset & $42(93)$ & $5(100)$ \\
\hline \multicolumn{3}{|l|}{ Durai on of use (months): } \\
\hline$<1$ & $21(47)$ & $1(20)$ \\
\hline $1-2$ & $16(36)$ & $1(20)$ \\
\hline$\geqslant 3$ & $8(18)$ & $3(60)$ \\
\hline \multicolumn{3}{|l|}{ Drug used: } \\
\hline Methimazole & $33(73)$ & $3(60)$ \\
\hline Propylthiouracil & $8(18)$ & $1(20)$ \\
\hline Carbimazole & $4(9)$ & $1(20)$ \\
\hline \multicolumn{3}{|c|}{ Aplastic anaemia } \\
\hline Drug used daily & $3(75)$ & $5(100)$ \\
\hline Drug last used on day before admission & $3(75)$ & $5(100)$ \\
\hline \multicolumn{3}{|l|}{ Duration of use (years): } \\
\hline$<1$ & $2(50)$ & $1(20)$ \\
\hline$\geqslant 1$ & $1(25)$ & $4(80)$ \\
\hline Unknown & $1(25)$ & \\
\hline \multicolumn{3}{|l|}{ Drug used: } \\
\hline Methimazole & $3(75)$ & $3(60)$ \\
\hline Carbimazole & $1(25)$ & $1(20)$ \\
\hline Propylthiouracil & 0 & $1(20)$ \\
\hline
\end{tabular}

cases $(82 \%)$ and two controls $(40 \%)$ took antithyroid drugs for less than three months. The antithyroid drug used by most cases and controls was methimazole. Each of the three drugs was used more commonly by the cases. Because of the small numbers of controls taking drugs statistical comparisons between the drugs were not made. 
Eight (18\%) patients with agranulocytosis using antithyroid drugs were men and $37(82 \%)$ were women; one $(20 \%)$ control was a man and four $(80 \%)$ were women. Their mean ages were 50.4 years (SD 16.9) for patients with agranulocytosis and $46 \cdot 0(19 \cdot 3)$ for the controls; the median was 48 years for both groups. There was one death $(2 \%)$ in the cases taking antithyroid drugs and nine (4\%) among the 217 patients not taking the drugs.

\section{APLASTIC ANAEMIA}

Four $(3 \%)$ patients with aplastic anaemia and five controls $(0 \cdot 2 \%)$ had been exposed to antithyroid drugs during the period 29-180 days before admission to hospital. The estimate of relative risk associated with use of antithyroid drugs compared with no use during that period was $9 \cdot 2(95 \%$ confidence interval $1 \cdot 8$ to 47$)$, and the proportion of cases due to antithyroid drugs was $3 \%$. Because of sparse data we did not estimate the excess risk.

The table also shows the characteristics of use of antithyroid drugs among the patients with aplastic anaemia and controls. Three cases (75\%) and all the controls took antithyroid drugs daily and continued up to the day of admission. Drugs were taken for less than one year in two cases $(50 \%)$ and one control $(20 \%)$. Methimazole was the drug taken most commonly. None of the cases had taken propylthiouracil.

Three of the four cases and four of the five controls taking antithyroid drugs were women. The median age of cases was 51 and of controls 46 years. After two years of follow up none of the patients with aplastic anaemia who used antithyroid drugs had died.

\section{Discussion}

Both thyroid disease and its treatment are known to affect the haemopoietic system. Untreated hyperthyroidism is often accompanied by mild leucopenia. ${ }^{18}$ Transient leucopenia (white cell count $<4 \times 10^{9} / 1$ ) is a common side effect of treatment with antithyroid drugs and occurs in up to $12 \%$ of adults taking such drugs. ${ }^{19}$ It is relatively benign and often remains undetected if regular blood counts are not made. Agranulocytosis, however, is a severe condition usually presenting as an acute infection; it is sometimes fatal and needs to be treated in hospital.

Antithyroid drugs may affect the immune system indirectly by inhibiting thyroid cell activity. ${ }^{20}$ Use of antithyroid drugs is accompanied by pronounced changes in the proportions of activated $T$ helperlike and $T$ suppressor-like cells. ${ }^{21}$ Several studies have shown that agranulocytosis induced by antithyroid drugs is an autoimmune phenomenon in which lymphocytes and circulating antibodies to neutrophils have been sensitised to the drugs..$^{81122}$

Agranulocytosis has been estimated to occur among groups of users of antithyroid drugs with a frequency of $0.5 \%$ to $1 \%,{ }^{3.5}$ but the incidence over time has not been reported. These estimates have been obtained from reports in which agranulocytosis has not been defined by specific criteria. Including cases of less pronounced leucopenia or granulocytopenia would have led to overestimates of the incidence because they are much more common than agranulocytosis. Furthermore, users of antithyroid drugs who developed agranulocytosis might have been preferentially included in these studies, also resulting in overestimation of the incidence.

In the present study, based on the relative risk, the proportion of cases due to antithyroid drugs, and the average weekly incidence of agranulocytosis, we estimate that about six people per million exposed to antithyroid drugs in a one week period would develop agranulocytosis. Thus, if the risk is assumed to remain constant in each succeeding week of treatment, the risk associated with use for one year would be about three per 10000 drug users, an assumption that may not be justified as most reported cases and $82 \%$ of cases in our study developed during the first three months of drug use. ${ }^{23}$ The actual risk for one year of treatment could therefore be even less. It should be noted that the estimated excess risk was based on a relative risk that, although highly significant, had a wide confidence interval owing to the small number of controls using antithyroid drugs. Despite its instability the estimate shows the order of magnitude of the excess risk.

Some published reports have suggested that the risk of agranulocytosis increases in relation to the dose of methimazole and propylthiouracil. ${ }^{23}{ }^{24}$ Information on dosage was not obtained in our study, but we found no evidence that the relative risk increased with longer use of antithyroid drugs.

The age and sex distributions of the patients exposed to antithyroid drugs are consistent with other reports. The ratio of women to men was 5:1 and the mean age was 50.4 (SD 16.9) years, both similar to those in a series of 50 cases described by Cooper (sex ratio 7:1; mean age 50.6 (16) years). ${ }^{25}$ Cooper found a significant difference in age between people who had been exposed to antithyroid drugs and developed agranulocytosis and those who did not. Because of the design of our study we could not examine age as an independent risk factor. Cooper reported one death among the 50 cases he examined. Our study had one death among the 45 exposed cases, which was not greatly different from the death rate of $4 \%$ among the 217 patients not exposed to antithyroid drugs. No information was obtained on the use of antithyroid drugs by the 23 patients who died before they could be interviewed, and the comparison may not be accurate.

Aplastic anaemia due to use of antithyroid drugs is rare, and the few reports of cases all relate risk of the dyscrasia to dosage. ${ }^{26-30}$ We could not evaluate this possibility because we did not obtain information on dosage, and the small number of drug users did not permit an examination of the duration of use of antithyroid drugs. Because of our limited data, the estimate of relative risk should be considered to represent no more than a positive association that is unlikely to be due to chance. The data were too sparse to justify estimation of the excess risk for use of antithyroid drugs, but clearly that risk is very low.

The effects of other drugs and other risk factors were controlled simultaneously by multivariate analysis, and confounding is unlikely to explain our results. Information bias and bias due to misclassification are not of great concern: as antithyroid drugs are taken regularly for the treatment of a serious condition their use is likely to be remembered and to be reported equally well by cases and controls. As they are a well known cause of agranulocytosis and their use is monitored closely, it is possible that patients with clinically mild disease who received antithyroid drugs were enrolled selectively, which could have resulted in overestimation of the risk. On the other hand, such surveillance could have resulted in stopping treatment in patients with reduced white cell counts before they developed agranulocytosis. Selection bias among controls was minimal as almost all of those approached were interviewed, and all controls had conditions unrelated to the use of antithyroid or other drugs.

Our data replicate the well known association between antithyroid drugs and agranulocytosis. The quantitative estimate of the excess risk was calculated to be 6.3 per million users in a one week period, which is lower than previous estimates. We also found an increased risk of aplastic anaemia among users of antithyroid drugs. Though the data were too sparse to 
justify an estimation of the excess risk, the results suggest that antithyroid drugs may cause aplastic anaemia but also that the excess risk associated with their use is very low.

This study was sponsored by Hoechst AG, Frankfurt, Federal Republic of Germany. Partial support was also provided by the governments of Hungary and Bulgaria and by the Corporation of Swedish Pharmacies. The study is supervised by an honorary international advisory board, whose members are Sir Richard Doll (Oxford), Per Knut M Lunde (Oslo), and Sven Moeschlin (Solothurn); it would not have been possible without the cooperation of over 300 hospitals in the participating centres.

PARTICIPANTS in the study were as follows: Chairman: M Levy, Jerusalem; Coordination and data analysis (Slone Epidemiology Unit, Boston): D Slone (deceased), S Shapiro, D W Kaufman, T Anderson, L J Blair, P T Cook, J J Farrell, L F Gaetano, J P Kelly, R Matis, C L Wells; Investigators: J R Laporte, X Carne, Barcelona; $H$ Kewitz, Berlin; G Retsagi, S Hollan, Budapest; M Levy, Z Stern, Jerusalem; G Tognoni, Milan; V Vlahov, Sofia; B E Wiholm, M Keisu, Stockholm; H Heimpel, W Heit, Ulm; Data collection: L Ibanez, Barcelona; M Klein, C Scott, Berlin; E Czink, A Laszlo, Budapest; M Beiski, F Simon, Z Toledano, Jerusalem; C LaVecchia, P Liati, M G Zurlo, Milan; N Bacracheva, Sofia; K Jacobsen, M Sandberg, Stockholm; I Bayer, R Kreuzer, Ulm; Haematology review committee: H Heimpel, W Heit, Ulm; G Lambertenghi-Deliliers, Milan; A Polliack, Jerusalem.

1 MacKenzie CG, MacKenzie JB. Effect of sulfonamides and thioureas on the thyroid gland and basal metabolism. Endocrinology 1943;32:185-209.

2 Astwood EB, Sullivan J, Bissell A, et al. Action of certain sulfonamides and of thiourea upon the function of the thyroid gland of the rat. Endocrinology 1943;32:210-25.

3 Trotter WR. The relative toxicity of antithyroid drugs. Fournal of New Drugs 1962;2:333-43.

4 Ahn YS, Yunis AA. Antithyroid therapy and leukocytes. In: Dimitrol NV, Nodine JH, eds. Drugs and hematologic reactions. New York: Grune and Stratton, 1974:249-59.

5 Rosove MH. Agranulocytosis and antithyroid drugs. West f Med 1977;126: 339-43.

6 International Agranulocytosis and Aplastic Anemia Study. The design of a study of the drug etiology of agranulocytosis and aplastic anemia. Eur $\mathcal{C}$ Clin Pharmacol 1983;24:833-6.

7 International Agranulocytosis and Aplastic Anemia Study. Risks of agranulocytosis and aplastic anemia: a first report of their relation to drug use with special reference to analgesics. $\mathcal{F} A M A 1986 ; 256: 1749-57$.

\title{
Gastrointestinal complications of dialysis related amyloidosis
}

\author{
E R Maher, S Hamilton Dutoit, R A Baillod, \\ P Sweny, J F Moorhead
}

Departments of

Nephrology and

Histopathology, Royal

Free Hospital and School

of Medicine, London

NW3 2QG

E R Maher, MRCP, registrar in

nephrology

$S$ Hamilton Dutoit,

MRCPATH, lecturer in

histopathology

R A Baillod, $\mathrm{MB}$, associate

specialist

P Sweny, FRCP, senior lecturer

in nephrology

J F Moorhead, FRCP,

consultant physician

Correspondence to:

Dr Maher, department of nephrology.
8 Guffy MM, Goeken NE, Burns CP. Granulocytotoxic antibodies in a patient with propylthiouracil-induced agranulocytosis. Arch Intern Med 1984;144: 1687-8.

9 Fibbe WE, Claas FH, Van der Star-Dijkstra W, Schaafsma MR, Meyboom $\mathrm{RH}$, Falkenburg JH. Agranulocytosis induced by propylthiouracil: evidence of a drug dependent antibody reacting with granulocytes, monocytes and haematopoietic progenitor cells. Br f Haematol 1986;64:363-73.

10 Bilezikian SB, Laleli Y, Tsan MF, et al. Immunological reactions involving leukocytes. III. Agranulocytosis induced by antithyroid drugs. fohns Hopkins Med f 1976;138:124-9.

1 Weitzman SA, Stossel TP. Drug-induced immunological neutropenia. Lancet $1978 ; \mathrm{i}: 1068-72$.

12 Armitage P. Statistical methods in medical research. New York: Wiley, 1971:380-4.

13 Miettinen OS. Proportion of disease caused or prevented by a given exposure, trait or intervention. Am f Epidemiol 1974;99:325-32.

14 Rothman KJ. Modern epidemiology. Boston: Little Brown, 1986:38-9.

15 Miettinen OS. Estimability and estimation in case referent studies. Am $\mathfrak{J}$ Epidemiol 1976;103:226-35.

16 Schlesselman JJ. Case-control studies: design, conduct, analysis. New York: Oxford University Press, 1982.

17 Shapiro S, Levy M. Metamizol: een honderdjarige treurnis. Ned Tijdschr Geneeskd 1987;131:1680-1

18 Irvine WJ, Wu FCW, Urbaniak SJ, et al. Peripheral blood leucocytes in thyrotoxicosis (Graves' disease) as studied by conventional light microscopy. Clin Exp Immunol 1977;27:216-21.

19 Cooper DS. Antithyroid drugs. N Engl J Med 1984;311:1353-62.

20 Volpe R, Karlsson FA, Jansson R, et al. Evidence that antithyroid drugs induce remissions in Graves' disease by modulating thyroid cellular activity. Clin Endocrinol (Oxf) 1986;25:453-62.

21 Totterman TH, Karlsson FA, Bengtsson M, Mendel-Hartvig I. Induction of circulating activated suppressor-like $T$ cells by methimazole therapy for Graves' disease. $N$ Engl F Med 1987;316:15-22.

22 Wall JR, Fang SL, Kuroki T, et al. In vitro immunoactivity to propylthiouracil, methimazole, and carbimazole in patients with Graves' disease: a possible methimazole, and carbimazole in patients with Graves' disease: a possible
cause of antithyroid drug-induced agranulocytosis. 7 Clin Endocrinol Metab cause of antithyr $1984 ; 58: 868-72$.

23 Wiberg JJ, Nuttall FQ. Methimazole toxicity from high doses. Ann Intern Med 1972;77:414-6.

24 Arneborn P, Palmblad J. Drug-induced neutropenia: a survey for Stockholm 1973-1978. Acta Med Scand 1982;212:289-92.

25 Cooper DS, Goldminz D, Levin AA, et al. Agranulocytosis associated with antithyroid drugs. Effects of patient age and drug dose. Ann Intern Med 1983;98:26-9.

26 Heimpel H, Heit W. Drug-induced aplastic anemia: clinical aspects. Clin Hematol 1980;9:641-62.

27 Martelo OJ, Katims RB, Yunis AA. Bone marrow aplasia following propylthiouracil therapy: report of a case with complete recovery. Arch Intem Med 1967;120:587-90.

28 Edell SL, Bartuska DG. Aplastic anemia secondary to methimazole: case report and review of hematologic side effects. $\mathcal{J}$ Am Med Wom Assoc 1975;30:412-3.

29 Aksoy M, Erdem S. Aplastic anaemia after propylthiouracil. Lancet 1968;i: 1379.

30 Swanson M, Cook R. Drugs, chemicals and blood dyscrasias. Hamilton, Illinois: Drug Intelligence Publications, 1977:634.

(Accepted 28 April 1988)

return to haemodialysis four months later. Carpal tunnel decompressions were performed in 1982 and 1983 when she also suffered a spontaneous fractured neck of femur as a result of a bone cyst. In 1983 she began to complain of bilateral shoulder pains and other features of dialysis arthropathy. Two years later she was admitted with melaena. Upper gastrointestinal endoscopy showed nothing abnormal and arteriography localised the bleeding site to mid-jejunum. At laparotomy $90 \mathrm{~cm}$ of jejunum including the apparent source of bleeding was resected. After operation there was no further bleeding and the patient continued with maintenance haemodialysis. Histologically the jejunum showed no abnormality apart from extensive amyloid deposition within submucosal blood vessels.

Case 2-This patient started haemodialysis in 1972 for rapidly progressive crescentic glomerulonephritis. In 1979 she received a renal transplant but this never functioned. In 1985 bilateral carpal tunnel decompression was performed and histological examination confirmed amyloid. The next year she complained of persistent diarrhoea. Results of barium studies were not diagnostic and she continued to have diarrhoea until she died six months later from disseminated renal cell carcinoma. Necropsy showed systemic amyloid deposition (primarily in association with blood vessels) in the heart, lungs, skin, subcutaneous fat, gut, muscle, synovium, kidneys, liver, adrenal, thyroid, and lymph nodes. There was extensive deposition of amyloid throughout the 